\title{
Melanoma de Canal Anal Simulando Doença Hemorroidária. Relato de Caso
}

\author{
Melanoma of the Anal Canal Simulating Hemorrhoidal Disease. Case Report
}

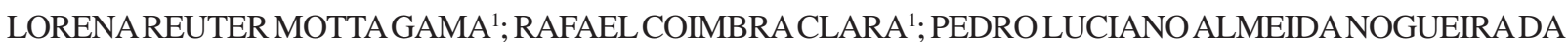 \\ GAMA $^{2}$; ALINE CRUZE SOUSAZAMBOM ${ }^{3} ;$ FLÁVIALEMOS MOURARIBEIRO $^{4}$; GIOVANNI JOSÉZUCOLOTO \\ LOUREIRO $^{5}$; LUCIANO PINTO NOGUEIRA DA GAMA ${ }^{5}$; ROSSINI CIPRIANO GAMA ${ }^{5}$
}

1. Acadêmica da Faculdade de Medicina de Caratinga, MG; Estagiária do Serviço de Coloproctologia do Vitória Apart Hospital; 2. Acadêmico da Faculdade de Medicina de Caratinga, MG; Estagiário do Serviço de Coloproctologia do Vitória Apart Hospital; 3. Acadêmico da Escola Superior de Ciências da Santa Casa de Misericórdia de Vitória, Emescam; Estagiário do Serviço de Coloproctologia do Vitória Apart Hospital; 4. Residente do Serviço de Coloproctologia do Vitória Apart Hospital; 5. Coloproctologista do Vitória Apart Hospital, ASBCP; 6. Coloproctologista do Vitória Apart Hospital, FSBCP; 7. Coloproctologista do Vitória Apart Hospital, TSBCP; 8. Coloproctologista do Vitória Apart Hospital, TSBCP.

\begin{abstract}
GAMA LRM; CLARA RC; GAMA PLAN; ZAMBOM ACS; RIBEIRO FLM; LOUREIRO GJZ; GAMA LPN; GAMA RC. Melanoma de Canal Anal Simulando Doença Hemorroidária. Relato de Caso. Rev bras Coloproct, 2010;30(3): 360-364.

RESUMO: O Melanoma Anorretal é um tumor maligno raro com a possibilidade de simular uma doença anorretal, tornando o diagnóstico difícil. Tem baixos índices de cura e elevados índices de mortalidade em curto prazo. Os autores descrevem um caso de melanoma de canal anal cuja interpretação por parte do paciente tratava-se de uma doença hemorroidária que exteriorizava às evacuações. Os autores fazem uma extensa revisão da literatura dando ênfase aos sintomas e a melhor opção terapêutica a ser instituída.
\end{abstract}

Descritores: Melanoma anorretal; câncer anorretal; doenças anais; melanoma; amputação abdominoperineal.

\section{OBJETIVO}

O objetivo deste trabalho é relatar um caso de Melanoma de Canal Anal atendido e tratado no Serviço de Coloproctologia do Vitória Apart Hospital - VAH, em janeiro de 2009, demonstrando a possibilidade deste tipo de tumor simular uma doença hemorroidária do terceiro para quarto grau.

\section{CONCLUSÃO}

Melanoma anorretal é uma entidade pouco freqüente e com prognóstico bastante reservado. A localização no canal anal da doença pode simular doenças anorretais inclusive com prolapso da lesão como foi observado neste caso. O diagnóstico e o tratamento cirúrgico realizados de forma precoce podem trazer uma maior sobrevida ao paciente, sendo importante que os pacientes sejam sempre bem examinados pelo proctologista.

\section{INTRODUÇÃO}

O Melanoma Anorretal é um tumor maligno e muito agressivo, responsável por $4 \%$ de todas as doenças malignas da região anal ${ }^{14}$. Embora seja incomum, a região anal é o terceiro local mais acometido pelo melanoma, depois do tipo cutâneo e ocular ${ }^{18}$. O termo

Trabalho realizado no Serviço de Coloproctologia do Vitória Apart Hospital, Vitória-ES.

$\overline{\text { Recebido em 27/08/2010 }}$

Aceito para publicação em 27/09/2010 
Melanoma define os tumores que apresentam melanócitos, que sintetizam ou depositam o pigmento melanina, que quando encontrados nas células malignas se tornam a base dos diagnósticos histológicos, porém não sendo sinal patognomônico desta doença ${ }^{3}$. Os melanócitos são derivados da crista embriológica neural que, durante o desenvolvimento fetal podem migrar para vários locais do corpo, principalmente a pele, olhos e superfícies mucosas. A faixa etária prevalente fica entre a sexta e oitava décadas de vida. Pode existir uma discreta predominância no sexo feminino, mas ainda é uma controvérsia na literatura ${ }^{18}$. Os sintomas mais comuns são dor local, sangramento às evacuações, alterações do hábito intestinal ou aparecimento de massa tumoral. Existem situações em virtude do crescimento da lesão delas poderem prolapsar durante o ato defecatório. O diagnóstico pode ser dificultado devido à sintomatologia poder simular doenças anorretais ${ }^{19}$, associado à raridade do tumor tornando o prognóstico bastante reservado. A sobrevida está intimamente ligada ao diagnóstico precoce da doença. A base do tratamento consiste no diagnóstico o mais precoce possível e na avaliação da disseminação metastática ${ }^{1}$. Não há um consenso na literatura sobre o tratamento cirúrgico, porém o procedimento de escolha varia desde a amputação abdominoperineal até mesmo uma excisão local ${ }^{21}$. Os tratamentos neoadjuvantes e adjuvantes têm benefícios limitados e nem sempre aumentam a sobrevida do paciente.

\section{RELATO DO CASO}

Paciente W.A.E, 71 anos, sexo masculino, branco, natural do ES, foi admitido no serviço de Coloproctologia do Vitória Apart Hospital em janeiro de 2009, com queixa principal de evacuação com sangue e muco, há dois anos. Relatava também tenesmo e prolapso hemorroidário que, no inicio reduzia espontaneamente, evoluindo para redução digital. Ao final de 2008 começou a apresentar exacerbação e maior freqüência dos sintomas e perda ponderal importante. Ao exame proctológico (toque) em janeiro de 2009, observou-se uma lesão vegetante, volumosa, endurecida e pouco fixa que prolapsava o canal anal, principalmente ao final das evacuações. Procedeu-se a anuscopia que mostrou um enchimento tumoral na luz do aparelho. Durante a Retossigmoidoscopia, identificamos como sendo uma lesão vegetante e hiperpigmentada que ocupava além do canal anal, o reto inferior. A biópsia dessa lesão foi duvidosa, sugerindo ser Adenocarcinoma pouco diferenciado infiltrando o reto inferior. $\mathrm{Na}$ Colonoscopia, foram encontrados quatro pólipos sésseis em cólon sigmóide, ângulo hepático e ceco, além de doença diverticular do cólon esquerdo. Realizado Polipectomia Endoscópica que mostrou na histopatologia tratar-se de Adenoma tubular de atipia de alto grau. O estudo imuno-histoquímico da biópsia do tumor do reto foi consistente com Melanoma Maligno (Marcadores positivos: Melan A, HMB-45 e Proteína S-100. FIGURAS 1 e 2). O estadiamento tomográfico do tórax mostrou-se normal e a do abdome mostrou tumoração infiltrativa e vegentante de reto inferior. A RNM da pelves demonstrou uma lesão infiltrativa e sólida no reto inferior, próxima a transição anorretal, determinando redução importante da luz do reto inferior, estendendo-se à gordura adjacente até próximo ao músculo elevador do ânus, além de linfonodos na gordura mesorretal e na cadeia dos vasos ilíacos externos. CEA 3.74. Não foi evidenciado nenhum outro possível foco do tumor, sugerindo este melanoma anorretal ser primário. Foi discutido com a equipe de oncologia sobre a indicação ou não de tratamento neo-adjuvante (quimioterapia e radioterapia), porém devido à baixa resposta desse tumor ao procedimento, optou-se pela ressecção cirúrgica como tratamento imediato. O paciente foi submetido à amputação abdominoperineal do reto (cirurgia de Miles) em fevereiro de 2009, com colostomia definitiva ( $F I$ GURA 3). O procedimento ocorreu sem complicações e o pós-operatório (P.O.) obteve boa evolução, recebendo alta hospitalar no $10^{\circ}$ dia. $\mathrm{O}$ anátomopatológico mostrou tratar-se de Melanoma Maligno Nodular, de canal anal na transição anorretal, pigmentada, estendendo-se até o músculo Pubo retal, com índice de Breslow no nível IV-V, e presença de metástase em 20 dos 22 linfonodos ressecados. Após um mês de P.O. apresentou lombociatalgia bilateral, sendo realizado PET-SCAN / CT e Cintilografia Óssea, onde foram evidenciadas reações osteogênicas, podendo ser processo degenerativo, porém não descartando a possibilidade de processo inflamatório devido à proximidade cirúrgica. Após 19 meses do diagnóstico e do tratamento, evoluiu para óbito, por falência de múltiplos órgãos e sistemas, devido a metástases generalizadas. 


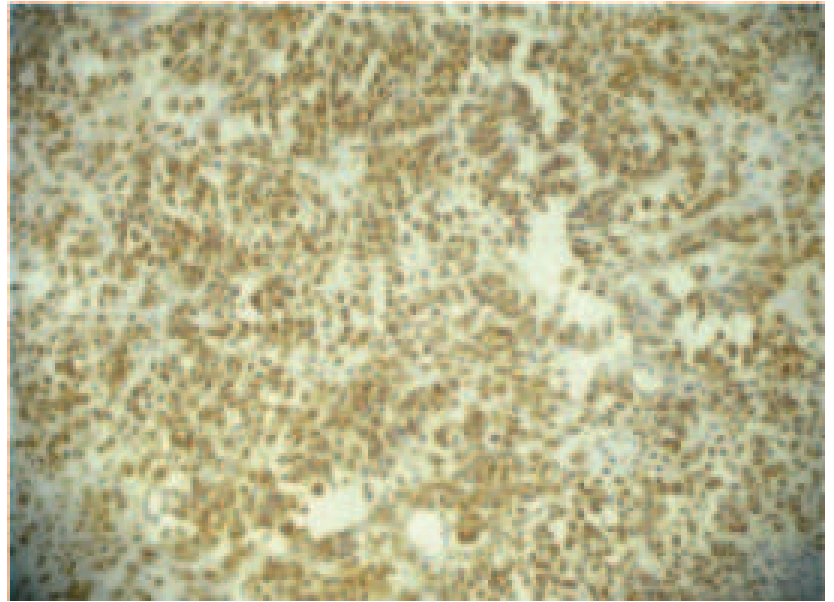

Figura 1 - Imuno-histoquímica: Marcador positivo Proteína S100.

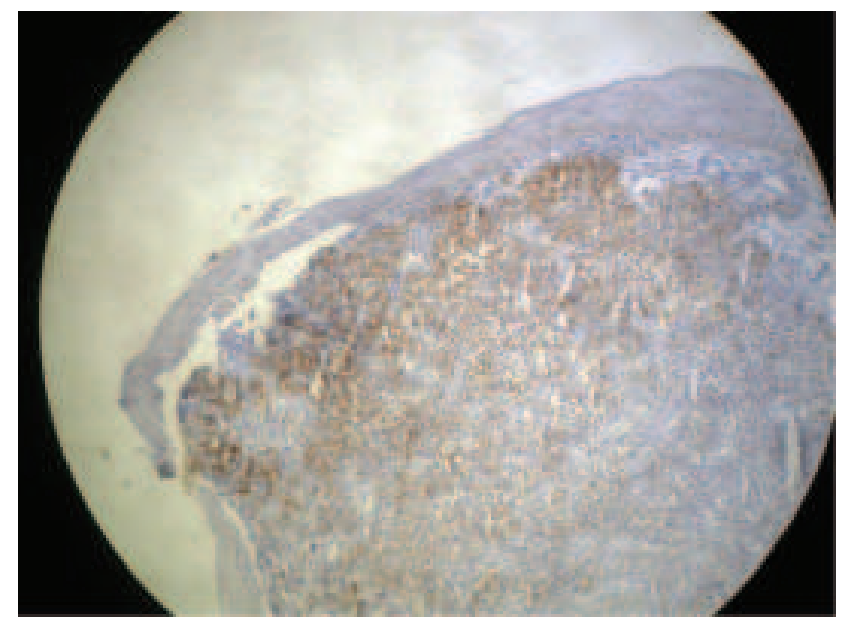

Figura 2 - Imuno-histoquímica: Marcador positivo Melan A.

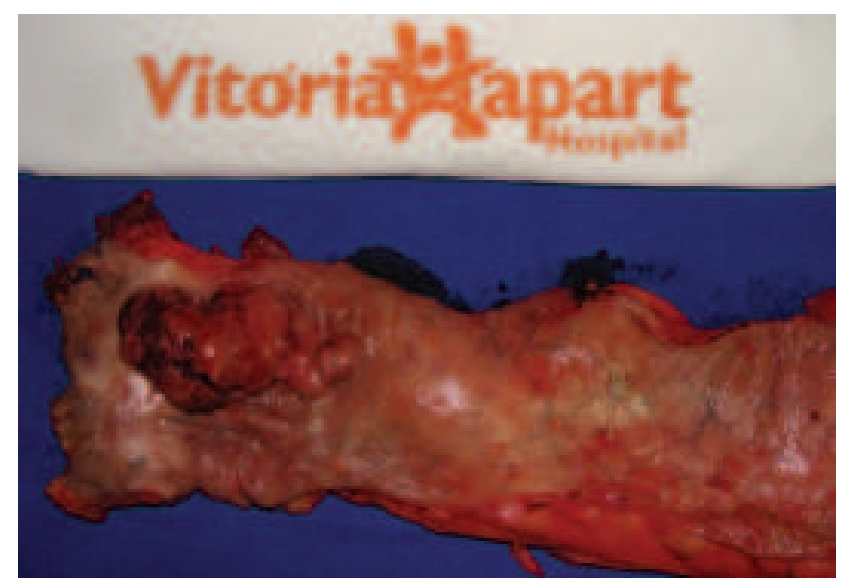

Figura 3 - Peça cirúrgica: produto de amputação abdominoperineal do reto mostrando volumosa tumoração de canal anal MELANOMA DE CANAL ANAL.

\section{DISCUSSÃO}

O melanoma maligno do reto é uma doença rara e muito agressiva. De acordo, com a Sociedade Americana de Câncer, estima-se que cerca de 62.480 novos casos de melanoma foram diagnosticados, nos Estados Unidos, em 2008 e que 8.420 morreram desta enfermidade. Os melanomas se originam a partir dos melanócitos encontrados em diversos pontos anatômicos como a epiderme, olhos, orofaringe, cavidade nasal, vagina, trato urinário e ânus. Melanomas cutâneos são de longe a forma mais comum da doença, respondendo a mais de $90 \%$ de todos os casos. Dos restantes, $5 \%$ correspondem a melanomas oculares, $2 \%$ para de origem desconhecida e $1 \%$ para melanomas de mucosa. Dentro deste subgrupo, o melanoma anorretal contribui com uma prevalência de $24 \%{ }^{17}$. Desde sua primeira publicação em 1857 até os dias atuais, existe cerca de 600 relatos de casos ao longo de todo o mundo, o que confirma a sua baixa incidência ${ }^{10}$. Os melanomas apresentam-se geralmente como lesões únicas, de tamanho bastante variável, podendo ser polipóides, sésseis ou mais freqüentemente pedunculados ou infiltrativos. Atinge, comumente, indivíduos entre a sexta e oitava década de vida e sua incidência é maior em brancos, sendo a doença rara em pessoas de outras raças ${ }^{1}$. Embora alguns relatos não demonstrem prevalência entre os sexos, alguns autores observam que há uma predominância no sexo feminino ${ }^{10}$. No entanto, deve-se levar em consideração o fato de que mulheres se submetem com mais frequiência a exames periódicos do períneo comparados aos homens, aumentando o número de diagnósticos nesse gênero ${ }^{17}$. Os sinais e sintomas, em geral, se assemelham a outras condições anorretais, tais como: dor abdominal e anal, sangramento retal, alteração de hábito intestinal, tenesmo e evacuação mucosanguinolenta ${ }^{19}$. Em caso de doença metastática, emagrecimento, anemia e fadiga podem estar presentes. $\mathrm{O}$ atraso no diagnóstico e a dificuldade em fazê-lo decorrem, comumente, da semelhança destas lesões com condições anorretais benignas, como a doença hemorroidária e pólipos e ao retardo em procurar auxílio médico. Contribui também o fato de que em cerca de $16 \%$ a $41 \%$ dos casos as lesões são amelanocíticas ${ }^{19}$. A disseminação pode ser hematogênica ou linfática e não guarda relação com o tamanho da lesão. Os principais sítios metastáticos por via hematogênica são fígado, pulmões, cérebro e os- 
sos. Quando ocorrem metástases linfáticas, elas localizam-se na cadeia inguinal, mesentérica e paraaórtica ${ }^{10}$. A disseminação também pode ocorrer localmente. Recomenda-se, com mais frequiência o método de Breslow para a classificação dos tumores, correlacionando a espessura do tumor à sua disseminação e prognóstico ${ }^{3}$. A avaliação anatomopatológica para diagnóstico é suficiente, porém em alguns casos faz-se necessário o uso da imuno-histoquímica para definição da lesão. A fim de complementar diagnóstico e estadiamento, utilizam-se exames de imagem como ultrasonografia endoanal para avaliar extensão e grau de invasão da lesão, além de presença ou não de gânglios metastáticos, tomografia computadorizada e ressonância magnética. Em relação ao tratamento ainda é assunto controverso. Excisão local e ressecção abdominoperineal são as propostas técnicas para ressecção cirúrgica do melanoma anorretal. Existem poucos estudos na literatura comparando estas técnicas, porém, em geral, não há diferença quanto à sobrevida global. Ganesh et al também demonstrou o uso da videolaparoscopia como opção a abordagem do melanoma anorretal, tendo como benefício a diminuição da morbidade, não se aplicando a tumores grandes e invasivos ${ }^{14}$. Dados na literatura sobre o efeito da radioterapia isoladamente ou em combinação com cirurgia são poucos e inconsistentes. Embora a radioterapia adjuvante não tenha resultado em melhora na sobrevida global, alguns efeitos no controle regional da doença foram demonstrados. Outras opções de terapia adjuvante incluem imunoterapia, braquiterapia e quimioterapia. A quimioterapia adjuvante não demonstrou benefício significativo na sobrevida ${ }^{14}$. Em relação ao prognóstico é muito ruim, havendo uma sobrevida média de aproximadamente 20 meses, sendo a cura muito rara. Na presença de metástase a sobrevida cai de $20 \%$ a $30 \%$ para $10 \%{ }^{16}$. Fatores associados com pior prognóstico incluem duração dos sintomas por mais de três meses, invasão perineural, presença de 10 linfonodos inguinais, melanoma amelanótico na histologia e classificação de Breslow demonstrando espessura do tumor e" $2 \mathrm{~mm}$.

ABSTRACT: Anorectal melanoma is a rare malignant tumor with the small chance of simulating anorectal disease, making the diagnosis hard. There is a low rate of cure and high rate of short term mortality. The authors describe a case of anal melanoma which by the patient's interpretation was hemorrhoid disease that exteriorized evacuations. The authors do an extensive evaluation of the literature, giving emphasis to the symptoms and the best therapeutic option to be used.

Key words: Anorectal melanoma, anorectal cancer, anal diseases, melanoma; abdominoperineal amputation

\section{REFERÊNCIAS}

1. Albino PA, Rossoni MD, Nunes BLBBP, Nossa FLC, Formiga GJS, Silva JH. Melanoma Maligno Anorretal - Relato de Três Casos e Revisão da Literatura. Rev bras Coloproct. 1998; 18(1): 30-33.

2. Bianchi A, Hidalgo LA, Muns R, Admella C, Suñol X. Melanoma Maligno Primario de Colon. Rev Esp Enferm Dig (Madrid). 2005; 97(12): 917-926.

3. Campos FG, Habr-Gama A, Silva JH, Ibrahim RE, Tuder R, Pinotti HW. Melanoma Maligno da Região Anorretal: Apresentação de Um Caso e Revisão da Literatura. Rev bras Coloproct. 1990; 10: 71-76.

4. Cruz GMG, Santana JL, Santana SKAA, Ferreira RMRS, Neves PM, Faria MNZ. Doenças Anais Concomitantes à Doença Hemorroidária: Revisão de 1.122 Pacientes. Rev bras Coloproct. 2006; 26(3): 369-376.

5. Cruz GMG, Santana JL, Santana SKAA, Constantino JRM, Chamone BC, Ferreira RMRS, et al. Exame Histopatológico em Espécimes de Doença Hemorroidária Operada - Revisão de 2.134 casos. Rev bras Coloproct. 2007; 27(3): 269-277.

6. Cruz GMG, Silva IG, Teixeira RG, Andrade Filho JS, Pena GPM

- Melanoma Anorretal. Apresentação de Três Casos e Revisão da literatura. Rev bras Coloproct. 1999; 19(3): 177-191.

7. Das P, Crane CH, Ajani JA. Current Treatment for localized and Carcinoma. Curr Opin Oncol. 2007; 19: 396-400.

8. Felz MW, Winburn GB, Kallab AM, Lee JR. Anal Melanoma: An Aggressive Malignancy Masquerading as Hemorrhoids. Southern Medical Journal. 2001; 94(9): 880-885.

9. Góes JRN, Fagundes JJ, Medeiros RR, Peres MAO, Chain EA, Leonardi LS. Tratamento do Melanoma Anorretal. Análise de Cinco Casos. Rev bras Coloproct. 1986; 6(3): 136-139.

10. Hossne RS, Prado RG, Bakonyi Neto A, Denardi Jr. E, Ferrari A. Melanoma Anorretal - Relato de Dois Casos e Revisão da Literatura. Rev bras Coloproct. 2007; 27(3): 317-321.

11. Mota OM, Machado MM, Silva O, Campoli PM. Melanoma Amelanótico Anorretal. Relato de Caso. Rev bras Coloproct. 1999; 19(1): 22-23. 
12. Müller CR, Mateus A, Oliveira SCV, Steckert JS, Silva NR, Carvalho LAR, et al. Melanoma Anorretal - Relato de Caso. Arq Catarinenses de Medicina. 2005; 34: 43-44.

13. Mutch MG, Roberts PL. Anal and Peri-anal Melanoma. Clinics in Colon and Rectal Surg. 2002; 15(4): 271-276.

14. Ramalingam G, Gan EY, Kutt-Sing W. Laparoscopic Abdominoperineal Resection for Anorectal Melanoma. ACase Report and Review of the Literature. Surg Laparosc Endosc Percutan Tech. 2009; 19(4): 149-151.

15. Rangel MF, Silva MVM, Pinto FSM, Fernandes MJC, Oliveira CVC, Simões JBR. Considerações Sobre o Tratamento Cirúrgico do Melanoma Maligno Anorretal. Rev Col Bras Cir. 1998; 25(5): 335-338.

16. Ribeiro PC, Carvalho GT, Santos AC, Randi Jr. R. Melanoma Anorretal. Rev bras Coloproct. 1982; 2(3): 96-98.

17. Row D, Weiser MR. Anorectal Melanoma. Clin Colon Rectal Surg. 2009; 22: 120-126.

18. Singer M, Mutch MG. Anal Melanoma. Clin Colon Rectal Surg. 2006; 19(2): 78-87.

19. Tagliolatto Jr. L, Muraro CAS, Muraro CLPM, Silva CEM, Lima Neto JR. Melanoma Maligno Anorretal - Relato de um Caso e Revisão da Literatura. Rev bras Coloproct. 1987; 7(4): 159-163.
20. Tanaka S, Ohta T, Fujimoto T, Makino Y, Murakami I. Endoscopic Mucosal Resection of Primary Anorectal Malignant Melanoma: A Case Report. Acta Med Okayama. 2008; 62(6): 421-424.

21. Van Schaik PM, Ernest MF, Meijer HA, Bosscha K. Melanoma of the rectum: A rare entity. World J Gastroenterol. 2008; 14(10): 1633-1635.

22. Vieria SC, Soares MM, Branco AGOC, Silva FM, Pessoa VS, Vasconcelos JF. Melanoma Anorretal: Relato de Um Caso. Rev bras Coloproct. 1995; 15(1): 29-30.

23. Yeh JJ, Shia J, Hwu WJ, Busam KJ, Paty PB, Guillem JG, et al. The Role of Abdominoperineal Resection as Surgical Therapy for Anorectal Melanoma. Ann of Surg. 2006; 244(6): 1012-1017.

Endereço para correspondência: ROSSINI CIPRIANO GAMA

Rodovia BR 101 norte, KM 02

Carapina, Serra-ES

CEP: 29161-900.

Telefones/FAX: 3348-5464 / 3348-5466

e-mail:rcgama@unimedvitoria.com.br 